\title{
Blockchain Corda-based IoT-Oriented Information- Sharing System for Agricultural Worker Physical Movement Data with Multiple Sensor Unit
}

\author{
Shinji Kawakura and Ryosuke Shibasaki
}

\begin{abstract}
In this study, we attempted to develop and implement a blockchain Corda-based record sharing system for traditional agricultural (agri-) researchers, workers, and their managers. Corda differs from other domain and practical blockchain techniques (e.g., Bitcoin, Ethereum) with respect to its main aims and structural features. Corda-based network systems can transmit and share basic data concerning ledgers at banks or other financial societies and can be handled on common web browsers (e.g., Google Chrome or Microsoft Internet Explorer). This study consists of three phases: (1) designing and confirming the validity of the entire system, (2) constructing and tuning various minor system settings (e.g., programs or networking specifications), and (3) conducting experiments in indoor settings using hoe acceleration data obtained from previous research projects. The integrated system performed with an acceptable level of accuracy. However, it was extremely difficult to quantitatively present the accuracy data. We were unable to concretely show the success and error rates for the data transmitting and receiving, nor the examination operation time. We thus present the specific error content. Overall, the main error trends were (1) errors concerning the rather small transaction time-delay, (2) mistakes concerning the transaction data in the system, and (3) broken transaction data in the system. In particular, we could determine the transaction time delay according to the JavaScript operations and features by observing. We present experimental ranges for these time delays and other error types. Noting concerns concerning previous trials, we suggest practical applications of the proposed system. In short, we believe that our results are novel achievements in the fusion of agricultural informatics, statistics, and human dynamics. We believe that combining this data and other kinds of timeline data with blockchain-based technology and multiple sensors will improve not only agri-business and management, but also agri-skill and security.
\end{abstract}

Index Terms-Blockchain, Corda, information-sharing, multiple sensor unit.

\section{INTRODUCTION}

In recent years, agricultural (agri-) researchers and agriworkers have developed a wide range of automatic and mechanical techniques to improve the utilities, particularly combining informatics and electric engineering. For actual agri-management sites, obtaining and sharing real-time information is a crucial factor directly related to the success or failure of modern agri-management. Recent promising studies in diverse research and business-fields have investigated blockchain-based network or similar systems [18]. Kamilaris, Fonts, and Prenafeta-Boldv́ [1] presented the latest blockchain technology-based trends in agriculture and food supply fields, particularly with respect to food science and technology. Omar, et al. [2] and Cichosz, Stausholm, et al. [3] constructed and presented concrete examples of blockchain technology-based platform systems for health care [2], and practical treatments for diabetic patients [3]. Regarding blockchain for diverse vehicles, Liu, et al. constructed and presented blockchain-enabled security systems according to electric vehicle clouds and edge computing [4], and Cebe, et al. looked at a light-weight blockchain framework for forensic applications of networkconnected vehicles [5]. From the perspective of IoT-based blockchain security and integrity, $\mathrm{Yu}$, et al. presented blockchain-based solutions to enhance the security and privacy levels of the Internet of Things (IoT) [6]. Machado and Fröhlich verified IoT data integrity concerning cyberphysical systems using blockchain-based systems [7]. With respect to robotics, Strobel, et al. managed byzantine robots with blockchain technology for a swarm robotics decisionmaking scenario [8].

More closely related cases include past studies of the (mesh-shaped) network system for agri-research and business-based management [9-13]. Fukatsu, Kiura, and Hirafuji [9-10] designed, developed, and handled web-based sensor network systems. They used the systems with Field Servers for practical agricultural applications to obtain diverse data from real outdoor agri-fields, such as humidity, temperature, and soil components (e.g., nitrogen (N), phosphoric acid (H3PO4), and potassium (K)) [10]. Fukatsu and Nanseki [11] developed and executed a monitoring system using wearable devices with sensor networks, such as a head-mounted camera, for real farming operations. Additionally, Brun-Laguna, et al. demonstrated the PEACH IoT-based frost event prediction system for agri-fields with consideration of recent precision agriculture techniques [12]. Karim and Karim [13] constructed and managed a monitoring system using Web of Things (WoT) for precision agriculture.

Several studies have collected and analyzed diverse human or robot physical data [14-20]. Zhao, et al. executed and presented a model targeting agri-robotics based on an acceleration sensor [14]. Some research has employed deep learning [15-18]. For example, Orii, et al. recognized tactile textures using recent deep learning-based methodologies (convolutional neural networks (CNNs)) for time-series data of pressure and 6-axis acceleration sensors [15]. Dawar and Kehtarnavaz detected and recognized human continuous streaming actions using deep learning-based sensing fusions [16]. Jiang and Yin recognized human activities from wearable sensors using deep CNNs [17], similar to Hassan, et al. [18]. Kawakura, and Shibasaki built integrated wearable sensing and agri-technical teaching systems, using three-axis acceleration sensors and angular velocity sensors, and 
presented their statistics [19-20].

Considering the aforementioned literature, we developed and attempted to implement a block chain-based agri-records sharing system for traditional, physical agri-workers, and managers. In this trial, considering technical backgrounds and trends, we selected Corda, which was designed and constructed by R3 (which consists of more than 70 financial groups (R3CEV LLC Inc., New York, USA)). Corda is a promising infrastructure that allows the sharing of basic text and numerical data concerning ledgers at banks or other financial organizations.

We believe that almost-real-time data-sharing approaches for agricultural worker's physical movement and other physical information with blockchain-based technology and multiple sensors will improve agri-skills and enhance security levels in the future.

\section{METHOD}

\section{A. Outline}

This study consists of three phases: (1) designing and confirming the validity of the entire system, (2) constructing and tuning various minor system settings (e.g. programs, networking specifications), and (3) conducting experiments in indoor settings using hoe acceleration data obtained from previous research projects.

To inform phase 1 , we performed a literature review to identify achievements and similar research and tools. Then, we designed systems to be practically executed in outdoor cultivated land settings (farmlands). During this phase, we incorporated the opinions of farmers with about 10 years of agricultural experience (career). In phase 2, we built the system and verified the portions judged to be the essentials of the operation (Figs. 1-7). In phase 3, we performed the operation experiments in indoor settings. However, for methodological reasons, we could not experiment in outdoor environments that had sufficiently connected Wi-Fi. Therefore, we used the previously collected timeseries data for hoe vertical acceleration (Fig. 8-10). That is, instead of performing experiments in outdoor settings, we used the previously collected data.

In future work, we plan to operate the wholly-connected (scientifically integrated) system in real outdoor farmlands where we can utilize Wi-Fi with sufficient communication conditions.

\section{B. Blockchain-based Computing System}

In this trial, we selected and used Corda-based systems. Corda was designed and constructed by R3 (R3CEV LLC Inc., New York, USA). Specifically, Corda allows us to share basic text and numerical data concerning ledgers at banks or other financial organizations. R3 consists of more than 70 financial groups with main members including Barclays, Goldman Sachs, BBVA, J.P. Morgan, Morgan Stanley, Mitsubishi UFJ Financial Group, Mitsui-Sumitomo Bank, and SBI Holdings. Corda's techniques differ from other domain blockchain-based social fundamental techniques, such as Bitcoin Core, Lightning Network, Ethereum, Quorum, and Hyperledger Fabric. However, these techniques are mainly for sending and receiving data, and users must execute data-mining procedures (e.g., mining secret codes for virtual electric money like Bitcoin). By contrast, other techniques like Bitcoin are mainly for sending and receiving diverse digital data, and for data mining.

The key point of Corda is that it is based on informationsharing between the relevant parties only (persons or electric nodes). The system exchanges transactions with tags that have private codes for the relevant the parties concerning the connections of the "Dealings (data exchanging)". This setup makes the information sharing process much safer.

Therefore, unlike other blockchain infrastructures, Cordabased systems cannot broadcast transactions to outsiders, and they have other novel network characteristics. For example, a notary-based system prevents double dealings and ensures the uniqueness of a transaction.

A transaction hashed chain prevents later alterations and records written information in Unspent Transaction Output (UTXO) format. This format is one of the most important concepts in blockchain field. The name might seem a little confusing, however the concept itself is simple. The features strengthen the safety of the data in the system.

For Corda's transaction approach, we selected and executed using "State"; this means current conditions of the network. We can share the State object with a Java-based program. The State is unchangeable (un-overwritable). After we use it once, the system replaces it with a new one. The sequential flow is presented with Bitcoin's data model: the UTXO model. The real state is recorded on the distributed ledger Vault. UTXO consists of the State that makes information inputted as Input and the State that makes outputting information as Output. The State changed to Output condition will be reused as the incoming transaction's Input [21-25].

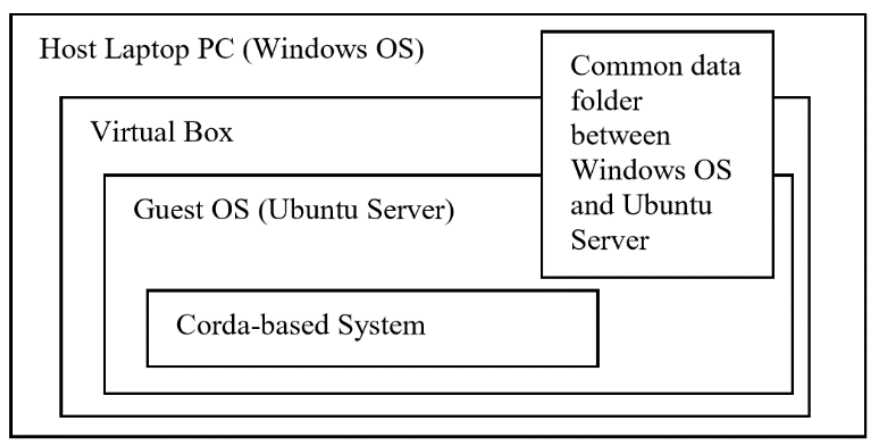

Fig. 2. Construction of the system in a laptop PC.

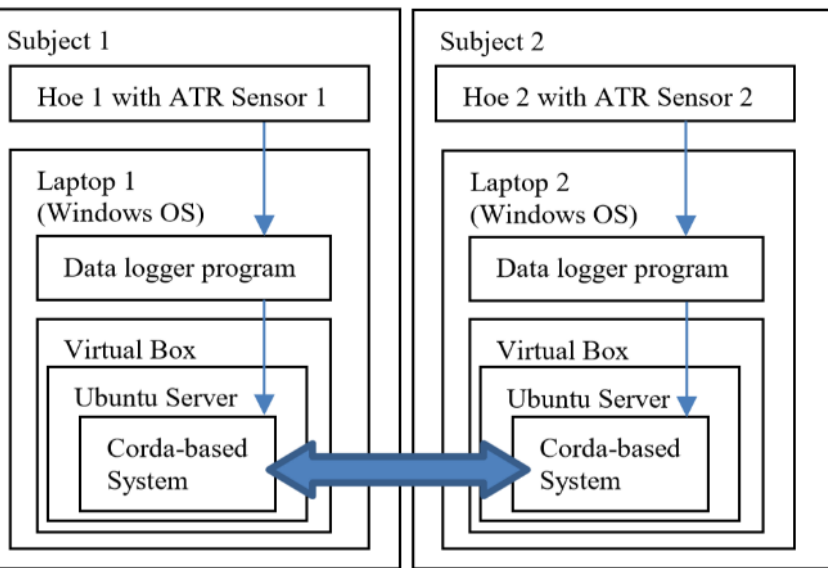

Fig. 1. Construction of the system between two laptop PCs. 


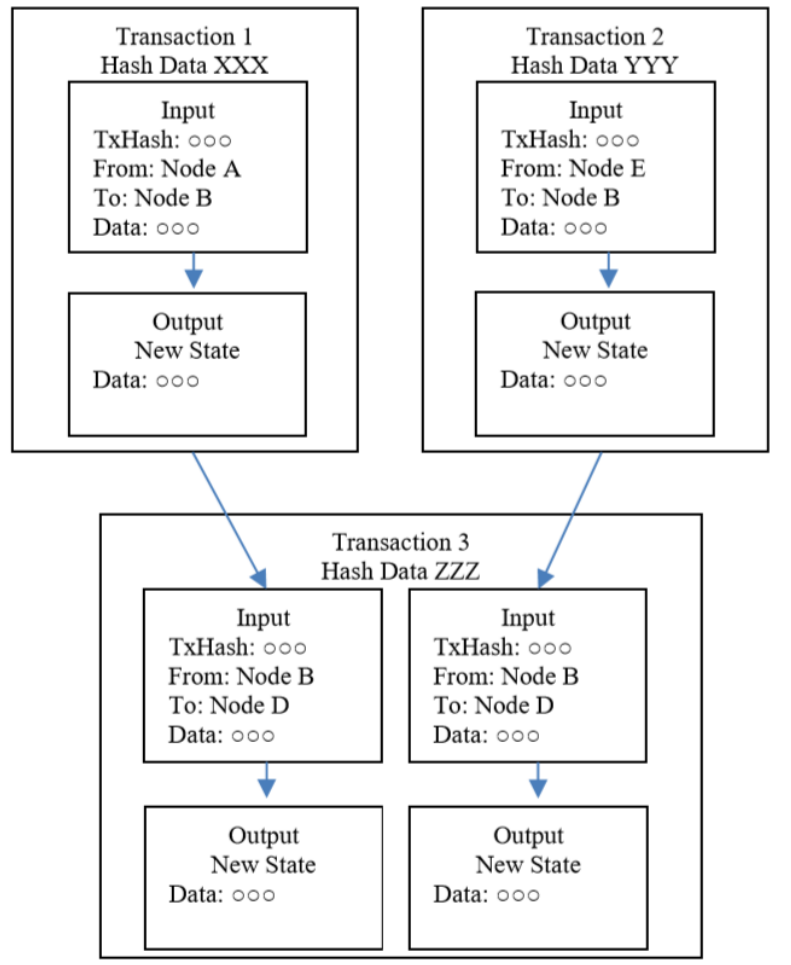

Fig. 3. The simplest construction for transacting with a Corda network: the UTXO-Model.

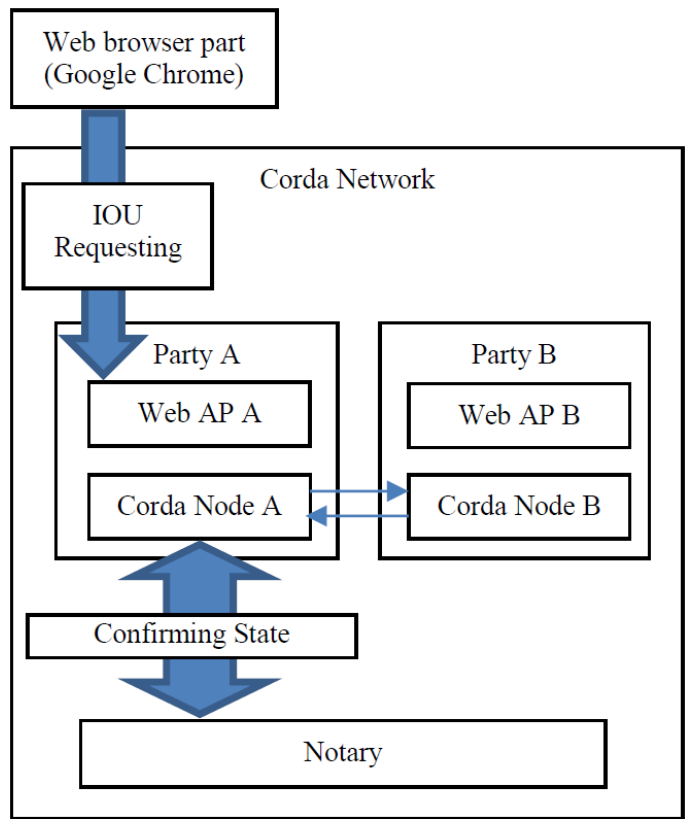

Fig. 4. Access to the Corda network from web browsers.

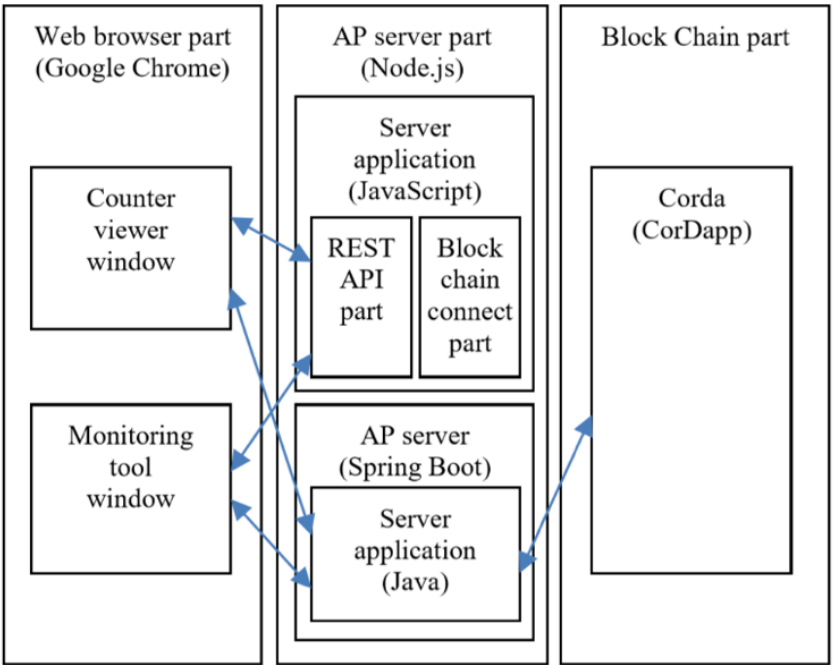

Fig. 5. Construction of the system in a laptop PC.

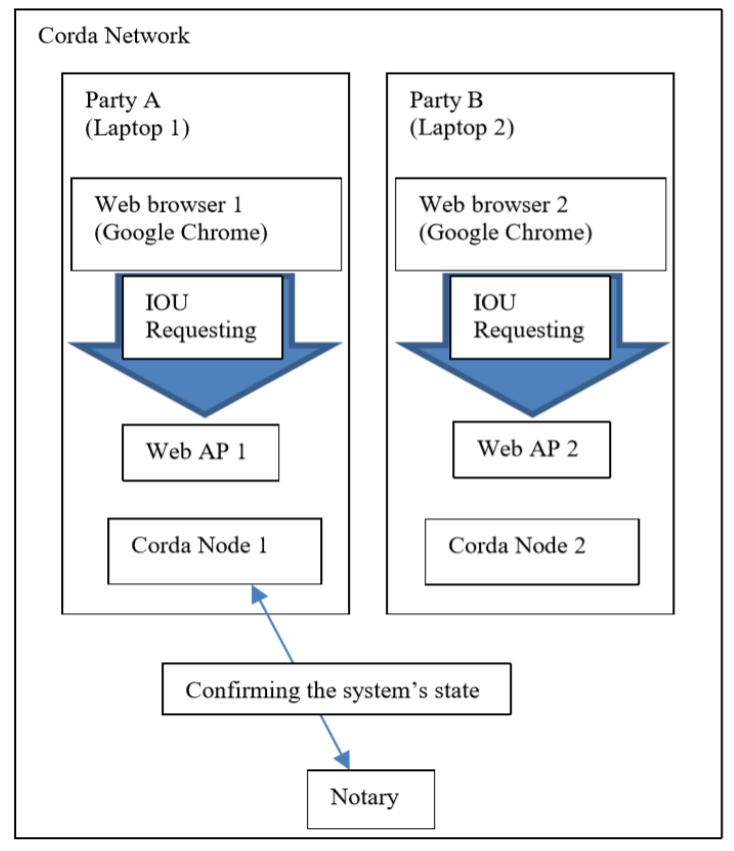

Fig. 6. Construction of the Corda network. 


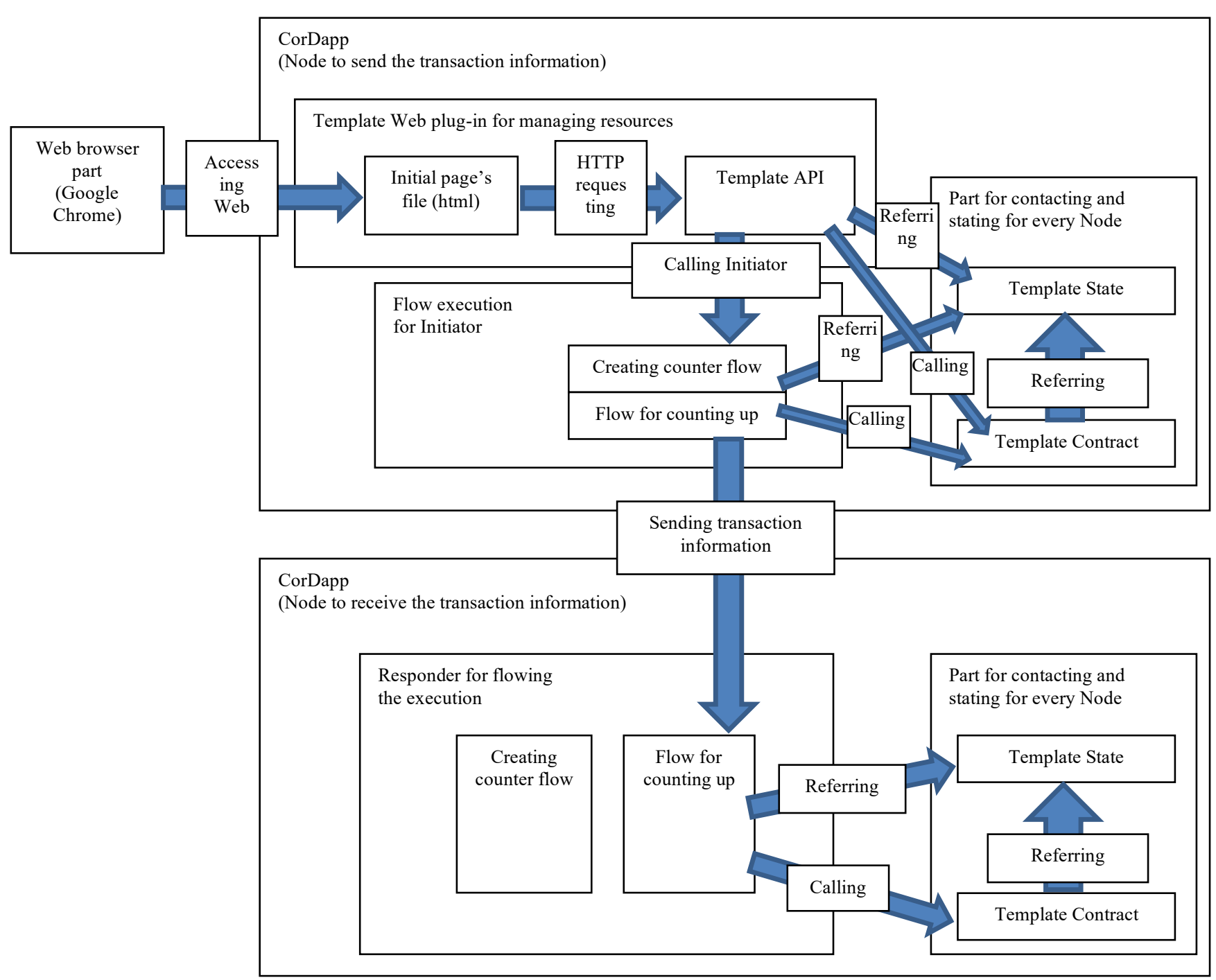

Fig. 7. Relationships of classes in the programs.

\section{Sensor-based System for subjects}

Various types of hoe are available for purchase globally, although these differ among districts, climates, and agricultural styles. In this study, we selected the KairyoKuwa hoe because it is popular in the Kantou region where the study was conducted. The specifications of the hoe include a weight of $1.65 \mathrm{~kg}$, a total length of $1200 \mathrm{~mm}$, and a head size of $120 \mathrm{~mm} \times 425 \mathrm{~mm}$. The shaft was made from light wood. The point where subjects grasped the hoe with their dominant hand was $300 \mathrm{~mm}$ from the head of the hoe. Subjects could grasp the tool anywhere with their other (nondominant) hand. For our target physical amount, we selected the relative (i.e., not global coordinates) vertical acceleration data as shown in Fig. 8. The authors used TSND121 multisensors (ATR-Promotions Inc., Japan) as shown in Fig. 9. The TSND121 module was attached at $150 \mathrm{~mm}$ from the grip hand on a hoe and connected with the blockchain Cordabased network, as presented in Fig. 10.

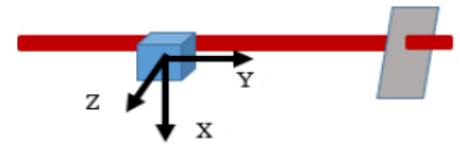

Fig. 8. The three axes of the acceleration sensor module attached to a hoe.

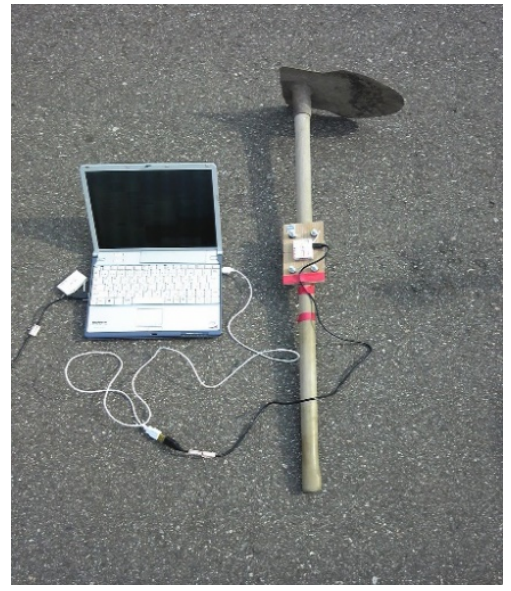

Fig. 9. Hoe connected to ATR integrated sensor unit and laptop PC. 


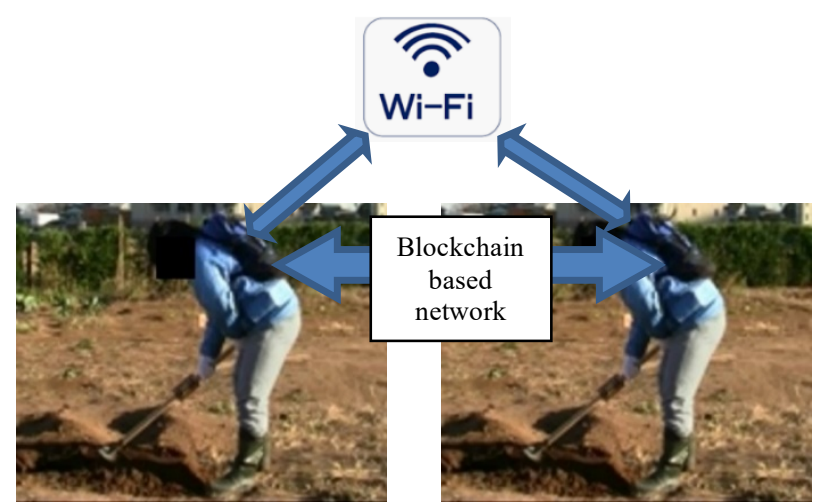

Fig. 10.I mage showing subjects equipped with the sensing system connected with blockchain-based network.

\section{Experimental Trial}

In phase 3, we conducted indoor experiments using previously accumulated timeline hoe acceleration data. We simulated the data as the information to share in the limited range of the blockchain Corda-based network. Future practical studies will test the system in areas of real farmlands (e.g., non-specific cultivated farms or rice fields).

\section{RESULT}

In this study, we designed, constructed, and executed the proposed system using previously obtained timeline acceleration data. This was an unprecedented example and with experimental content.

Although we applied the integrated system with a certain level of accuracy, it was extremely difficult to quantify the accuracy data. In this study, we do not present the success and error rates $(\%)$ for the data transmitting and receiving, nor the examination operation time, because we did not define the appropriate trial time ranges and the error data were rather random. We could not find past academic precedents for this approach. Thus, we aim to understand the main error trends: (1) errors concerning the rather small transaction time-delay, (2) mistakes concerning the transaction data in the system, and (3) broken transaction data in the system.

We could determine the transaction time delay according to the JavaScript operations and features by observing . However, from our experimental ranges for these time delays and other kinds of errors, we suggest that these errors are not due to characteristics of the Corda infrastructure.

\section{DiscUSSION}

The previous section presented the error tendencies of these systems in operation. In this study, we judged that it was likely impossible to present numerical sets of fixed quantitative data illustrating the experimental results. Therefore, we provided written examples of the confirmed errors. However, we plan to increase the number of subjects and nodes and to prolong the experimental time for future trials. Therefore, we should use recent and emerging big data and machine learning-based analysis technologies. Further, we should determine the accuracies of such systems qualitatively and quantitatively.

In summary, to achieve universality for these trial systems, future studies need to confirm the number of data and changes in the experimental environment.

\section{CONCLUSION}

We developed and tested various basic steps of our systems and methodologies by examining actual agri-workers' physical acceleration data with a hoe. In this research, we attempted to develop and implement a blockchain Cordabased system with consideration for systems and analyses found in other fields. We analyzed and verified the system to some extent. We have provided significant and practical suggestions for agricultural leaders, managers, and newcomers, and we believe that our results have largely achieved the fusion of agricultural informatics, statistics, and human dynamics. In future, we believe that combining agricultural worker physical movement data with blockchainbased technology and multiple sensors will improve agriskills and enhance security levels.

\section{ACKNOWLEDGMENT}

Our heartfelt appreciation goes to the members of Mitsui Fudosan Co., Ltd., Kashiwano-Ha Farm Inc., Kashiwa-shi, and The University of Tokyo who provided considered support, feedback, and comments.

\section{REFERENCES}

[1] A. Kamilaris, A. Fonts, and F. X. Prenafeta-Boldú, "The rise of blockchain technology in agriculture and food supply chains. Trends in Food Science \& Technology," vol. 91, pp. 640-652, January 2019.

[2] A. Al Omar, M. S. Rahman, A. Basu, and S. Kiyomoto, "Medibchain: A blockchain based privacy preserving platform for healthcare data," Proceedings of International conference on security, privacy and anonymity in computation, communication and storage, pp. 534-543, December 2017.

[3] S. L. Cichosz, M. N. Stausholm, T. Kronborg, P. Vestergaard, and O. Hejlesen, "How to use blockchain for diabetes health care data and access management: an operational concept," Journal of diabetes science and technology, vol. 13, no. 2, pp. 248-253, 2019.

[4] H. Liu, Y. Zhang, and T. Yang, "Blockchain-enabled security in electric vehicles cloud and edge computing," IEEE Network, vol.32, no. 3 , pp. $78-83$, January 2018 .

[5] M. Cebe, E. Erdin, K. Akkaya, H. Aksu, and S. Uluagac, "Block4forensic: An integrated lightweight blockchain framework for forensics applications of connected vehicles," IEEE Communications Magazine, vol. 56, no. 10, pp. 50-57, October 2018

[6] Y. Yu, Y. Li, J. Tian, and J. Liu, "Blockchain-based solutions to security and privacy issues in the internet of things," IEEE Wireless Communications, vol. 25, no.6, pp. 12-18, December 2018.

[7] C. Machado, and A. A. M. Fröhlich, "IoT data integrity verification for cyber-physical systems using blockchain," Proceedings of 2018 IEEE 21st International Symposium on Real-Time Distributed Computing (ISORC), pp. 83-90, May 2018.

[8] V. Strobel, E. Castelló Ferrer, and M. Dorigo, "Managing byzantine robots via blockchain technology in a swarm robotics collective decision making scenario," Proceedings of the 17th International Conference on Autonomous Agents and Multi Agent Systems, pp. 541549, July 2018.

[9] T. Fukatsu, T. Kiura, and M. Hirafuji, "A web-based sensor network system with distributed data processing approach via web application," Computer Standards \& Interfaces, vol. 33, no. 6, pp. 565-573, November 2011

[10] T. Fukatsu, and M. Hirafuji, "Web-based sensor network system Field Servers for practical agricultural applications," Proceedings of the 2014 International Workshop on Web Intelligence and Smart Sensing, pp. 1-8, September 2014.

[11] T. Fukatsu, and T. Nanseki, "Monitoring system for farming operations with wearable devices utilized sensor networks," Sensors, vol. 9, no. 8, pp. 6171-6184, July 2009.

[12] K. Brun-Laguna, A. L. Diedrichs, J. E. Chaar, D. Dujovne, J. C. Taffernaberry, G. Mercado, and T. Watteyne, "A demo of the 
PEACH IoT-based frost event prediction system for precision agriculture," Proceedings of 2016 13th Annual IEEE International Conference on Sensing, Communication, and Networking (SECON), pp. 1-3, June 2016

[13] F. Karim, and F. Karim, "Monitoring system using web of things in precision agriculture," Procedia Computer Science, vol. 110, pp. 402409, January 2017.

[14] S. Zhao, Z. Zhang, D. Xiao, and K. Xiao, "A Turning Model of Agricultural Robot Based on Acceleration Sensor," IFACPapersOnLine, vol. 49, no. 16, pp. 445-450, January 2016.

[15] H. Orii, S. Tsuji, T. Kouda, and T. Kohama, "Tactile texture recognition using convolutional neural networks for time-series data of pressure and 6-axis acceleration sensor," Proceedings of 2017 IEEE International Conference on Industrial Technology (ICIT), pp. 10761080, March 2017.

[16] N. Dawar, and N. Kehtarnavaz, "Action detection and recognition in continuous action streams by deep learning-based sensing fusion," IEEE Sensors Journal, vol. 18, no. 23, pp. 9660-9668, September 2018.

[17] W. Jiang, and Z. Yin, "Human activity recognition using wearable sensors by deep convolutional neural networks," Proceedings of the 23rd ACM international conference on Multimedia, pp. 1307-1310, July 2015.

[18] M. M. Hassan, M. Z. Uddin, A. Mohamed, and A. Almogren, “A robust human activity recognition system using smartphone sensors and deep learning," Future Generation Computer Systems, vol. 81, pp. 307-313, April 2018.

[19] S. Kawakura, and R. Shibasaki, "Timeline effects of vocal instructions from computer programs on agricultural technical teaching," Journal of Advanced Agricultural Technologies, vol. 1, no. 2, pp. 104-112, January 2014.

[20] S. Kawakura, and R. Shibasaki, "Statistical Analysis of Index Values Extracted from Outdoor Agricultural Workers Motion Data," Journal of Advanced Agricultural Technologies, vol. 1, no. 2, pp. 69-74, January 2014.

[21] N. Akazawa, "Python De Ugokashite Manabu Atarashii Block Chain No Kyokasho," Japan: FLOC Inc. November 2019.

[22] C. Kasaki, K. Shinohara, and H. Maruyama, "Block Chain Application Kaihatsu No Kyokasho,” Japan: Mainavi Inc. February 2018.

[23] J. Yamazaki, S. Ando, and S. Tanaka, "Block Chain Programing: Kaso tsuka Nyumon," Japan: KODANSHA Inc., August 2017.

[24] A. Watanabe, and Y. Matsumoto, "Hajimete No Block Chain Application; Ethereum Ni Yoru Smart Contract Kaihatsu Nyumon," Japan: SHOEISHA Inc., August 2017.

[25] T. Shimizu, K. Tamachi, and Y. Uenohara, "Block Chain No Kakushingijyutu $\sim$ Hyperledger Fabric Ni Yoru Application Kaihatsu," Japan: RicTelecom Inc., June 2018.

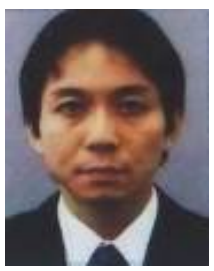

Shinji KAWAKURA Place of birth: Toyama Pref., Japan. Date of birth: July 14, 1978. Ph.D. in Environmentology, University of Tokyo, 2015, Bunkyo-ku, Tokyo, Japan. B.A. in Control System Engineering, Tokyo Institute of Technology, 2003, Meguro-ku, Tokyo, Japan. M.A. in Human-Factor Engineering, Tokyo Institute of Technology, 2005, Meguro-ku, Tokyo, Japan.

Career: Systems engineering, research for private companies. Development and verification of sensing systems for outdoor agricultural workers.

Dr. KAWAKURA, Department of Technology Management for Innovation at the University of Tokyo/Bunkyo-ku, Tokyo, Japan. Committee member of ICEAE and ICBIP.

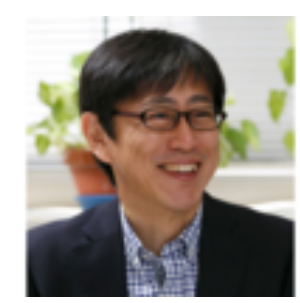

Ryosuke SHIBASAKI Place of birth: Fukuoka. Pref. Japan. Date of birth: March 1, 1958. Dr. in Engineering, the University of Tokyo, 1987, Bunkyo-ku, Tokyo, Japan. B.A in Engineering, the University of Tokyo, 1980, Bunkyo-ku, Tokyo, Japan. M.A. in Engineering, the University of Tokyo, 1982, Bunkyo-ku, Tokyo, Japan.

Career: Professor at the Center for Spatial Information Science, University of Tokyo.

Dr. SHIBASAKI, Center for Spatial Information Science (CSIS), The University of Tokyo, Meguro-ku, Tokyo, Japan, and Department of SocioCultural and Socio-Physical Environmental Studies, The University of Tokyo/Kashiwa-shi, Chiba, Japan. 